Annales Geophysicae (2004) 22: 537-548 (C) European Geosciences Union 2004

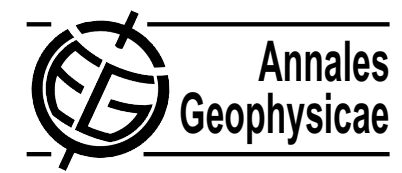

\title{
Auroral streamers: characteristics of associated precipitation, convection and field-aligned currents
}

\author{
V. A. Sergeev ${ }^{1}$, K. Liou ${ }^{2}$, P. T. Newell ${ }^{2}$, S.-I. Ohtani ${ }^{2}$, M. R. Hairston ${ }^{3}$, and F. Rich ${ }^{4}$ \\ ${ }^{1}$ Institute of Physics, St. Petersburg State University, Ulyanovskaya 1, Petrodvoretz, 198504, St. Petersburg, Russia \\ ${ }^{2}$ The Johns Hopkins University Applied Physics Laboratory, Laurel, Maryland, 20723, USA \\ ${ }^{3}$ Center for Space Sciences, University of Texas at Dallas, USA \\ ${ }^{4}$ AFRL, Hanscom Air Force Base, MA 01731, USA
}

Received: 14 February 2003 - Revised: 4 July 2003 - Accepted: 22 July 2003 - Published: 1 January 2004

\begin{abstract}
During the long-duration steady convection activity on 11 December 1998, the development of a few dozen auroral streamers was monitored by Polar UVI instrument in the dark northern nightside ionosphere. On many occasions the DMSP spacecraft crossed the streamer-conjugate regions over the sunlit southern auroral oval, permitting the investigation of the characteristics of ion and electron precipitation, ionospheric convection and field-aligned currents associated with the streamers. We confirm the conjugacy of streamer-associated precipitation, as well as their association with ionospheric plasma streams having a substantial equatorward convection component. The observations display two basic types of streamer-associated precipitation. In its polewardmost half, the streamer-associated (field-aligned) accelerated electron precipitation coincides with the strong $\left(\geq 2-7 \mu \mathrm{A} / \mathrm{m}^{2}\right)$ upward field-aligned currents on the westward flank of the convection stream, sometimes accompanied by enhanced proton precipitation in the adjacent region. In the equatorward portion of the streamer, the enhanced precipitation includes both electrons and protons, often without indication of field-aligned acceleration. Most of these characteristics are consistent with the model describing the generation of the streamer by the narrow plasma bubbles (bursty bulk flows) which are contained on dipolarized field lines in the plasma sheet, although the mapping is strongly distorted which makes it difficult to quantitatively interprete the ionospheric image. The convective streams in the ionosphere, when well-resolved, had the maximal convection speeds $\sim 0.5-1 \mathrm{~km} / \mathrm{s}$, total field-aligned currents of a few tenths of MA, thicknesses of a few hundreds km and a potential drop of a few $\mathrm{kV}$ across the stream. However, this might represent only a small part of the associated flux transport in the equatorial plasma sheet.
\end{abstract}

Correspondence to: V. Sergeev

(victor@geo.phys.spbu.ru)
Key words. Ionosphere (electric fiels and currents). Magnetospheric physics (aurroal phenomena; energetic particles, precipitating)

\section{Introduction}

Statistically, the transient bursty bulk flows (BBFs) provide the largest contribution to the plasma and magnetic flux transport in the plasma sheet (Angelopoulos et al., 1992; Baumjohann, 1993). Many of these high speed flows have a small cross-tail scale (a few Re), although the individual narrow plasma stream is capable of carrying a magnetic flux transport approaching $>60 \mathrm{kV}$, which is comparable to the global rate of magnetic flux circulation in the magnetosphere (Sergeev et al., 2000). Development of the BBFs is difficult to study in situ with a single (or a few closely spaced) spacecraft.

Recently, a close relationship between the fast bursty plasma sheet flows (BBFs) and auroral activations was confirmed observationally for a wide range of activity and for different types of transient auroral structures, including the localized auroral brightenings and pseudobreakups, and north-south auroras (or auroral streamers) (Fairfield et al., 1999; Ieda et al., 2001; Nakamura et al., 2001b; Sergeev et al., 2000, 2001; Lyons et al., 1999). Not only was a close temporal-spatial relationship found for auroras and isolated BBF events (including both tailward flow bursts corresponding to the plasmoids, Ieda et al., 2001 and earthward flows near the center of an expanded plasma sheet, Nakamura et al., 2001a, b), but the absence of perpendicular (cross-B) plasma flows in the plasma sheet in the absence of the auroral activations was also shown (Ieda et al., 2003). This close relationship suggests the attractive possibility of using the auroral activations as a tool for monitoring the high-speed plasma sheet flows if their spatial relationship and the mechanism 
generating the precipitation can be established. As of now, is not clear, as there exist several possibilities of generating the electron precipitation, as well as a number of factors which influence the outcome (see, e.g. Sergeev, 2002). The mechanisms may include (A) direct precipitation from the fast plasma stream; (B) field-aligned electron acceleration in the upward field-aligned current generated by the plasma stream, and $(\mathrm{C})$ acceleration by the field-aligned electric field generated by the difference of ion and electron pitch-angle distributions in the stream (e.g. Serizawa and Sato, 1984). Observationally these three mechanisms should differ in the relationship between the precipitation and field-aligned currents (FACs, which are important in mechanism B but play no role in mechanisms $\mathrm{A}, \mathrm{C}$ ), as well as in the relationship between the electron and proton acceleration and precipitation (here A differs from $\mathrm{C}$ ). Therefore, a study of the ion and electron precipitation and its relationship to the field-aligned currents provides a way to test which mechanism (and in which conditions) could be responsible for the precipitation from the fast flow region.

As already mentioned, the different types of transient localized auroral forms are observed in association with the BBFs. Among them one type, the auroral streamers, has the most obvious connection to the bursty bulk flows. A streamer can be best defined (see, e.g. a discussion in Sergeev et al., 2001) as a transient, narrow structure initiated in the poleward oval and propagating towards the equatorial oval boundary. In terms of plasma sheet origin, such dynamics and orientation (not always exactly north-south) implies a development of some narrow plasma structure in the earthward direction from the distant tail toward the inner magnetosphere. Not only is the general dynamics similar, but a close temporal and spatial association between these two phenomena has been established in case studies (Sergeev et al., 2000; Nakamura et al., 2001a, b).

There were a few previous attempts to probe the streamerassociated convection and FAC with ground-based (magnetic, radar and optical) observations recently summarized by Amm and Kauristie (2002). The spatial association of an auroral streamer with the the upward FAC was confirmed, as well as their closure by the downward FAC to the east of the streamer. However, only a couple of cases were actually analysed, and both the streamer identification and a search for associated convection patterns was hampered by the limited field-of-view of the ground-based instruments. Therefore, many important questions concerning the character of the precipitation (especially ion precipitation), its relationship with the convection structure, and field-aligned currents still remain open.

These questions could be better addressed with the precipitation, electric and magnetic fields probed simultaneously by the low altitude spacecraft. This is the purpose of our study in which we analyse a dozen streamer crossings made by three DMSP spacecraft that occured while their dynamics were monitored by the Polar UVI instrument.

\section{Observations}

\subsection{Instrumentation and event description}

The SSJ4 spectrometer at the DMSP spacecraft (at $850 \mathrm{~km}$ altitude) provides the measurements of precipitated ion and electron fluxes in 19 energy channels from $32 \mathrm{eV}$ and $30 \mathrm{keV}$ (in the following the ions are presumed to be the protons). We used three-component magnetic field measurements at 1-s time resolution (Rich et al., 1985), with the IGRF magnetic field contribution subtracted. The data are displayed in a local coordinate system with $X$ pointing along the spacecraft velocity, the $Z$-component pointing radial outward, and the $Y$-component completing the triad. In the Southern Hemisphere (where all DMSP data come from in our study) the orientation of the axes is schematically shown in the upper left corner of Fig. 1. The plasma flow components across the spacecraft trajectory $\left(V_{y}\right.$ and $V_{z}$ ) were measured by the driftmeter, whereas the convection component along the trajectory $\left(V_{x}\right)$ comes from the retarding plasma analyzer (RPA) (see Boyle et al. (1997) for more details concerning the processing and measurements). Here we used the convection flow data at 4-s time resolution. The RPA measurements are usually noisy as compared to the drift meter data, but for our purposes the $V_{y}$ component was more important, since in our cases it was mostly aligned along the streamer (see a scheme in Fig. 1) and was suitable for detecting the associated convection component, as well as to compute the potential drop of the convection jet.

A crucial part of our study is global imaging of the auroral streamers made by the Polar UVI instrument. On 11 December 1998 this instrument provided high time-resolution images of auroral behaviour in the northern auroral oval with good continuous coverage of its nightside part for $\sim 10 \mathrm{~h}$ between 01:00 and 12:00 UT. A complete sequence of images (in two alternating LBHL and LBHS bands at 36-s time resolution, e.g. Liou et al., 1997) is repeated every $3 \mathrm{~min}$.

The time interval of interest occurred in the middle of a long period of activity which had started on the previous day and continued throughout the day of interest, 11 December 1998. During the first $12 \mathrm{~h}$ the $K_{p}$ index was 4 to $5+, D_{s} t$ declined from $-40 \mathrm{nT}$ to $-60 \mathrm{nT}$, and the $A E$ index varied between 400 and $800 \mathrm{nT}$. According to the measurements at the Wind spacecraft (at $[47 ;-19 ;-25]$ Re GSM at 06:00 UT) and IMP8 spacecraft (at [26; 4; 13] Re), available via the CDAWeb site, the dynamic pressure varied between 2 and $6 \mathrm{nPa}$ and the IMF was basically southward with the brief northward excursions at around 03:40 and 06:20 UT. The activity during this time rarely displayed strong largescale enhancements in both the auroras and auroral electrojet which are typical for the substorms. An exception was a substorm starting at $\sim 08: 20$ UT and possible substorm-like events at around 06:00 UT. At other times the activity displayed the characteristics of so-called steady convection including a lot of localized fluctuations in electrojet currents and transient auroral features (see, e.g. Sergeev et al., 1996a, 2001; Lyons et al., 2002). The most remarkable among the 


\section{Conjugate Observations of Auroral Streamer (Type I)}
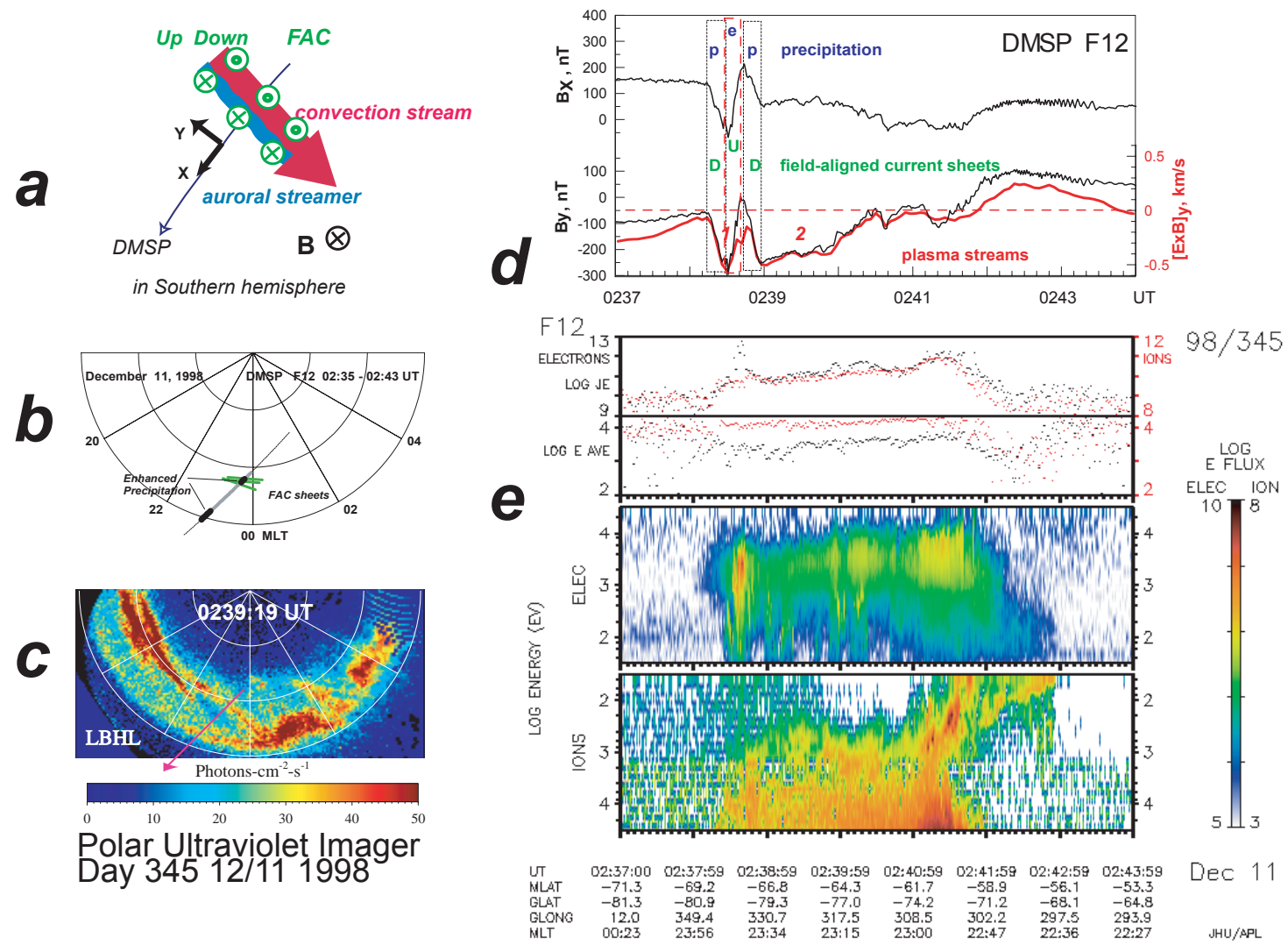

Fig. 1. Scheme of spacecraft crossing in the Southern Hemisphere through the auroral streamer, convection jet and associated field-aligned current sheets (a) and a summary of DMSP F12 observations for the crossing of the auroral streamer at 02:38 UT, including: (b) DMSP trajectory in the Southern Hemisphere with hatched regions of strong precipitation and positions/orientations of the planar FAC sheets (shown by green lines); (c) Polar UVI images in the Northern Hemisphere, the pink arrow shows approximately the mapped DMSP trajectory; (d) summary of transverse magnetic variations and convection flow measured by DMSP F12; (e) traces of proton and electron energy fluxes and average energies together with electron and proton spectrograms.

latter was the auroral streamer activity: about three dozen well-defined streamers developing from the poleward boundary of a wide oval toward its equatorward edge have been identified in the imager data during this half-day. Most of them (with a few exceptions) have been inclined from NW to $\mathrm{SE}$ and they started in the pre-midnight hours and ended in the midnight-post-midnight equatorward diffuse oval.

Due to the specifics of the DMSP orbits, all auroral zone crossings near midnight occurred in the Southern Hemisphere. Continuous streamer activity allowed us to identify a large number of crossings of three DMSP spacecraft (F12, F13 and F14) over the region magnetically conjugate to the auroral streamers. They will be studied below. Although the streamers embedded in the auroral oval are expected to be on the closed field lines of the plasma sheet, one should not ignore possible variations of the positions of magnetically conjugate points (mapped along the field lines). The differences are particularly expected due to the large dipole tilt angle existing at the time of these crossing (close to its extremal value $-34^{\circ}$ ), which results in a $2^{\circ}$ to $4^{\circ}$ (depending on equa- torial distance and model) poleward shift in ACGM latitude of the northern conjugate point with respect to its latitude in the Southern Hemisphere. The streamer-related precipitation appeared to display different characteristics. We attempted to classify them into two groups, types I and II, whose examples are presented in the next sections. After presenting a summary of events we then discuss the appearance and interpretation of these differences.

\subsection{Examples of observations: Type I events}

We start presenting our cases with a single distinct streamer which makes associating the streamers from the two hemispheres unambiguous. Figure 1 presents a case where the streamer connecting the poleward oval boundary and equatorward oval was crossed in its polewardmost part. According to the DMSP F12 spectrogram, the most intense electron precipitation (with energy flux approaching $10^{13} \mathrm{eV} / \mathrm{cm}^{2} \mathrm{sr}$ ) was observed in the narrow structure located $\sim 1^{\circ}$ equatorward from the polar cap boundary. Intense precipitation of 

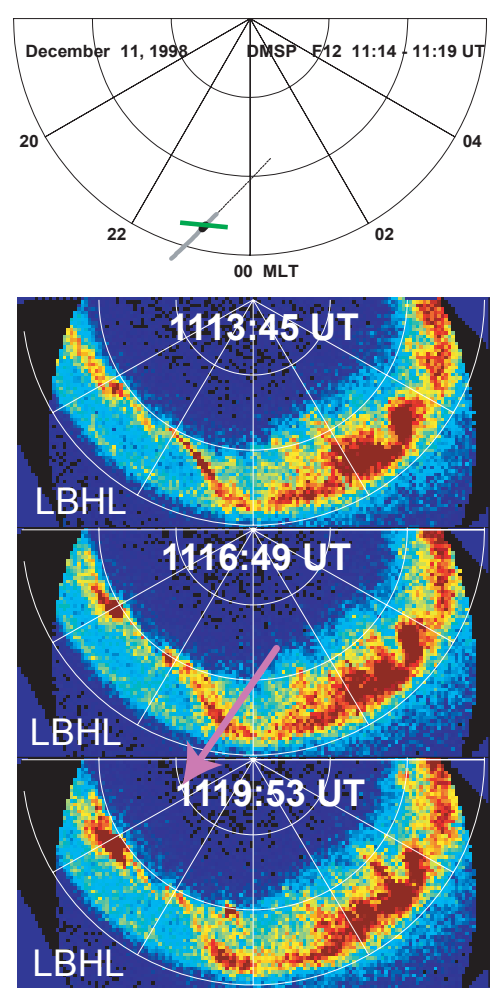
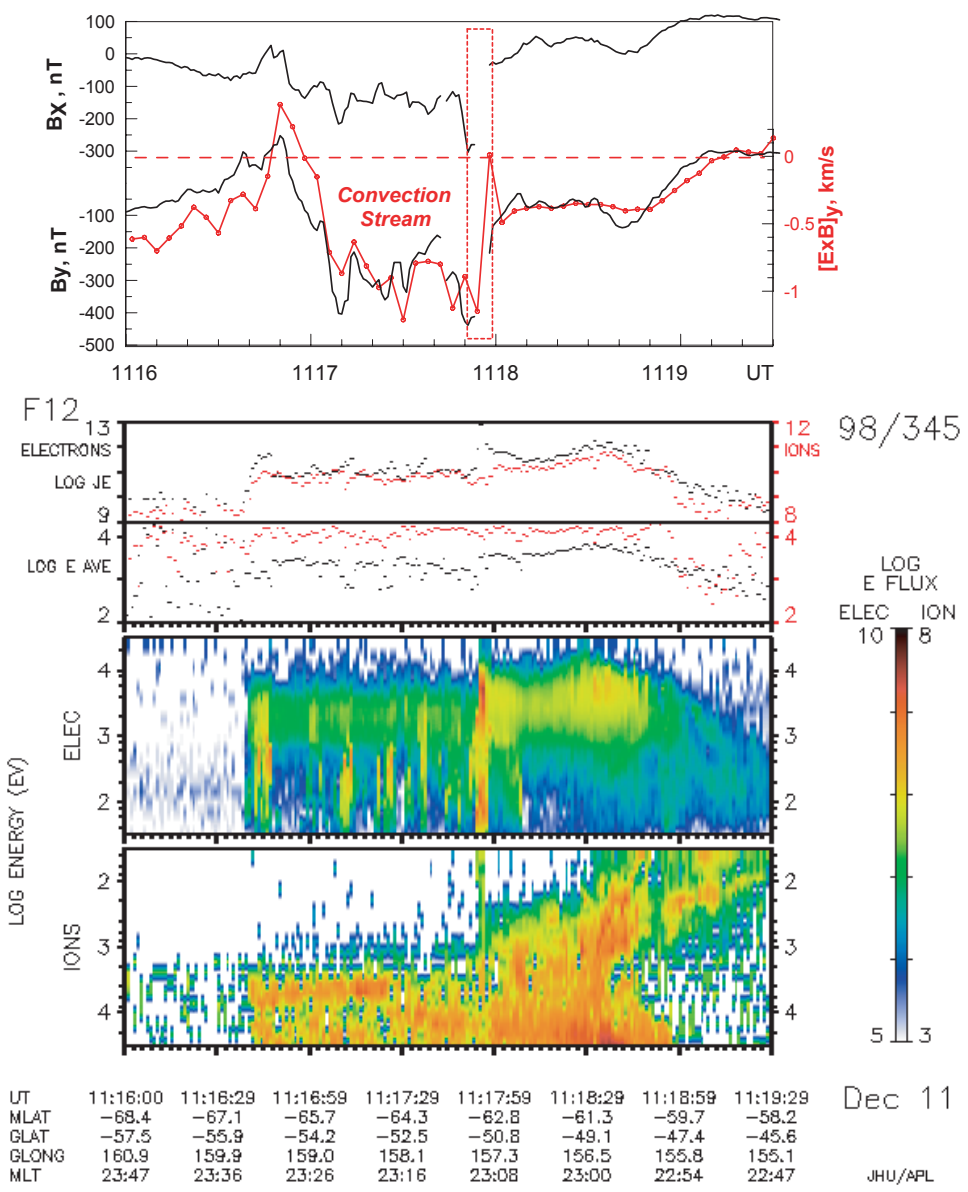

Fig. 2. Summary of DMSP F12 observations during the streamer crossing at 11:18 UT.

both protons and electrons, more widespread and diffuse, was also observed in the equatorward part of the southern oval. To facilitate comparison with Polar data, these enhanced precipitation regions are marked on the DMSP trajectory shown in the same scale and same coordinates (ACGM latitude versus Magnetic Local Time) above the Polar UVI image. Comparison confirms the conjugacy if a reasonable shift ( $\sim 2^{\circ}$ poleward and $\sim 0.5 \mathrm{~h}$ MLT westward) of conjugate points in the Northern Hemisphere is applied. Two intense precipitation regions are, therefore, identified to be the mappings of the auroral streamer (narrow polewardmost intense precipitation of field-aligned accelerated electrons) and of the diffuse equatorward oval seen in Polar images.

Another important feature of this traversal is a sharply defined pattern of cross-B magnetic field variations. Near the streamer it shows a pattern characteristic for a triple sheet of the field-aligned current (downward-upward-downward). The planar geometry is suggested by a strong correlation between $D B_{x}$ and $D B_{y}$ field components (correlation coefficient $\mathrm{R}=0.87$ for 43 points covering all three sheets), their orientation (also shown on the F12 trajectory in Fig. 1) is consistent with the streamer orientation. The strongest FAC density $\left(j_{\|}>5 \mu \mathrm{A} / \mathrm{m}^{2}\right)$ was observed in the upward current sheet which coincided with the narrow intense precipitation of field-aligned accelerated electrons (its 10-s duration gives $L_{x} \sim 70 \mathrm{~km}$ scale size which is the upper estimate of the actual width of upward FAC and precipitation structure). The proton precipitation is depressed inside the electron precipitation structure. These are the characteristics of the type 1 streamer-related precipitation.

The convection flow measurements come from different instruments. For our goals the key component is $V_{y}$ which characterizes the convection flow along the streamer, the enhanced negative $V_{y}$ is expected in the case of equatorward plasma streaming. Indeed this is what was observed, although the event is more complicated as the enhanced equatorward convection forms two streams situated on both sides of the strong electron precipitation and upward FA current. A notable feature is a nice correlation between $D B_{y}$ and $V_{y}$ which particularly confirms the good quality of the $V_{y}$ measurements and will be used thereafter to estimate the coupling efficiency. (By contrast the $V_{x}$ measurements were rather noisy, displayed large data gaps, so they are omitted here.)

A very similar auroral pattern was observed during the DMSP F12 crossing displayed in Fig. 2. Here the narrow active streamer was the brightest aurora seen by Polar UVI along the DMSP trajectory. Accordingly, the 

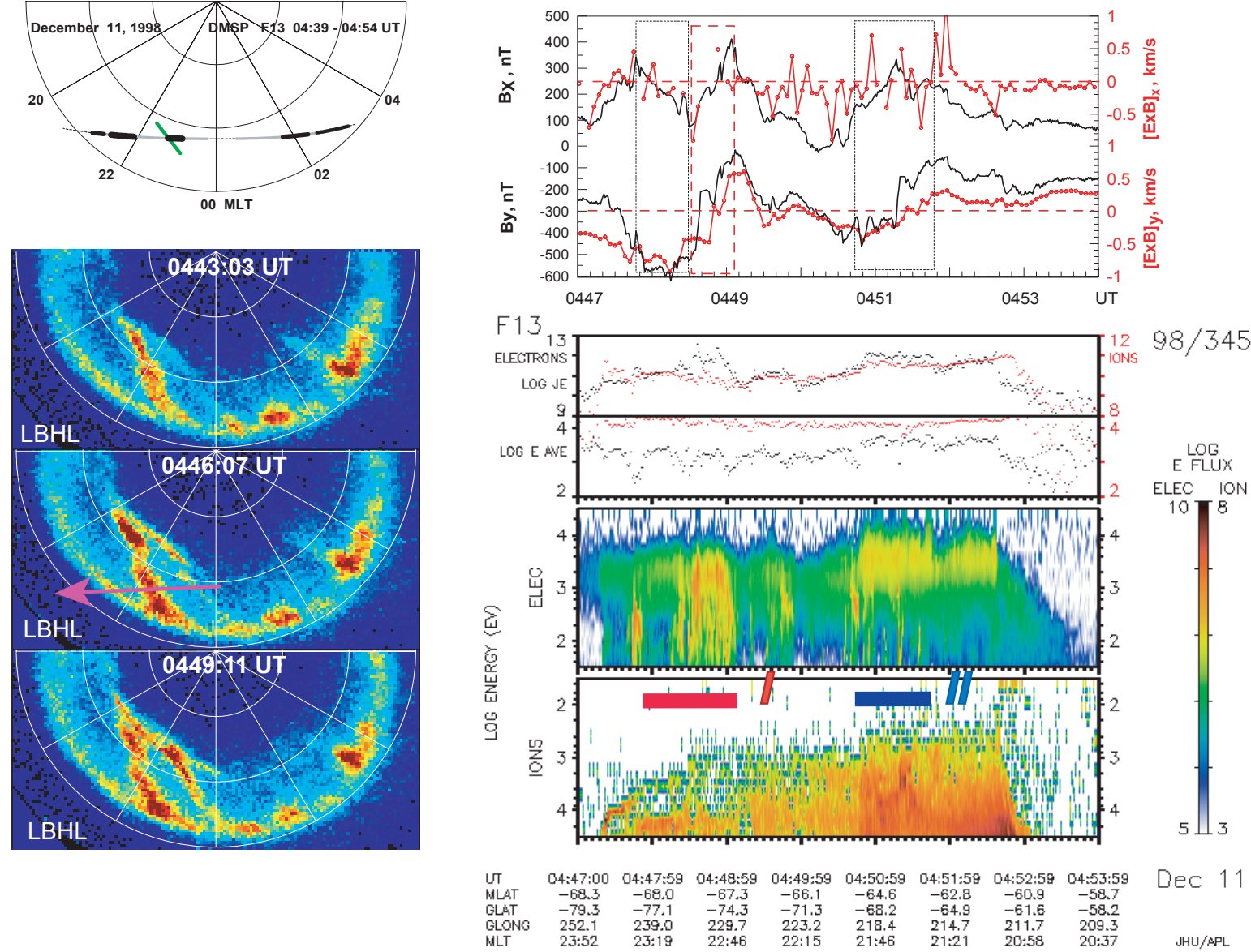

Fig. 3. Summary of observations during the streamer crossing by the DMSP F13 at 04:48 UT.

DMSP spectrogram shows the strongest intense (EF exceeding $10^{13} \mathrm{eV} / \mathrm{cm}^{2} \mathrm{sr}$ ), narrow ( $\Delta t \sim 6 \mathrm{~s}$ gives $\left.L_{x} \sim 40 \mathrm{~km}\right)$ precipitation at the corresponding location. Associated with it is a depressed proton precipitation, so it also belongs to the Type 1 category. Its association with very intense FAC $\left(j_{\|}>7 \mu \mathrm{A} / \mathrm{m}^{2}\right.$, with some data gaps) is also very clear. As distinct from the previous case, this streamer is observed at the dusk flank of a single, powerful stream of equatorward convection, which is rather wide $\left(L_{x} \sim 350 \mathrm{~km}\right)$ and strong $\left(V_{y}>1 \mathrm{~km} / \mathrm{s}\right)$. Such a pattern is consistent with the scheme shown in Fig. 1. ( $V_{x}$ has a data gap over the convection stream region and is not shown.) The DMSP encountered this streamer in the middle of the active auroral oval, which was $\sim 7^{\circ}$ wide.

In Fig. 3 the DMSP F13 satellite crossed two streamers which belong to different categories in our nomenclature. The first crossing (at 04:48:30-04:49:00 UT, close to the polar cap boundary near midnight) has the already known properties: the intense electron precipitation (with signatures of field-aligned acceleration) coincides with the region of upward FAC (having the properties of a planar sheet oriented along the streamer), which is located at the dusk flank of the equatorward convection (here both convection components are shown). According to Polar observations this was a crossing of the newly-born streamer. It was relatively wide ( $L_{x} \sim 250 \mathrm{~km}$ corresponding to $\Delta t=37 \mathrm{~s}$ ). The proton precipitation was rather depressed inside the streamer but it was enhanced between 04:47:50 and 04:48:30 UT, that is in the more dawn/poleward region inside of the stream of equatorward convection where the signatures of (less intense) downward FAC could also be noticed.

\subsection{Examples of observations: Type II events}

According to the global images from the Polar spacecraft, the next crossing of the auroral streamer along the DMSP trajectory in Fig. 3 is expected near 22:00 MLT meridian in the middle of the oval; this was a mature streamer born more than $10 \mathrm{~min}$ before. We can associate it with the enhanced precipitation of both electrons and protons, which sharply increased at 04:50:45 UT, continued for 60-s and smoothly joined diffuse precipitation region in the equatorward oval. In fact, the characteristics of this type II streamer-related precipitation are similar to those of diffuse equatorward precipitation and could be distinguished from the latter one only because the former was a bit more intense. Such properties remind us the so-called "dispersionless injection" events (Newell and Meng, 1987), although the latter ones have been associated with the substorms. As compared to the magnetic variations, 

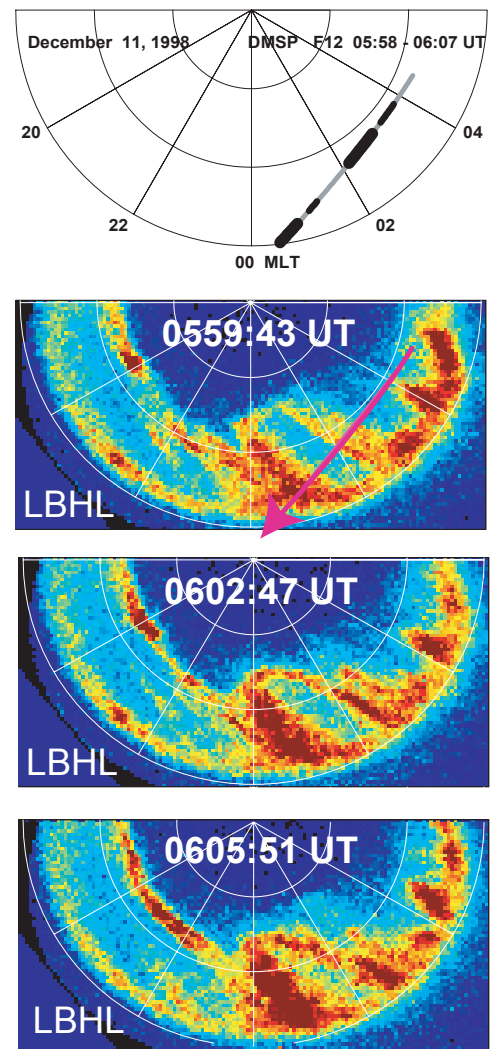
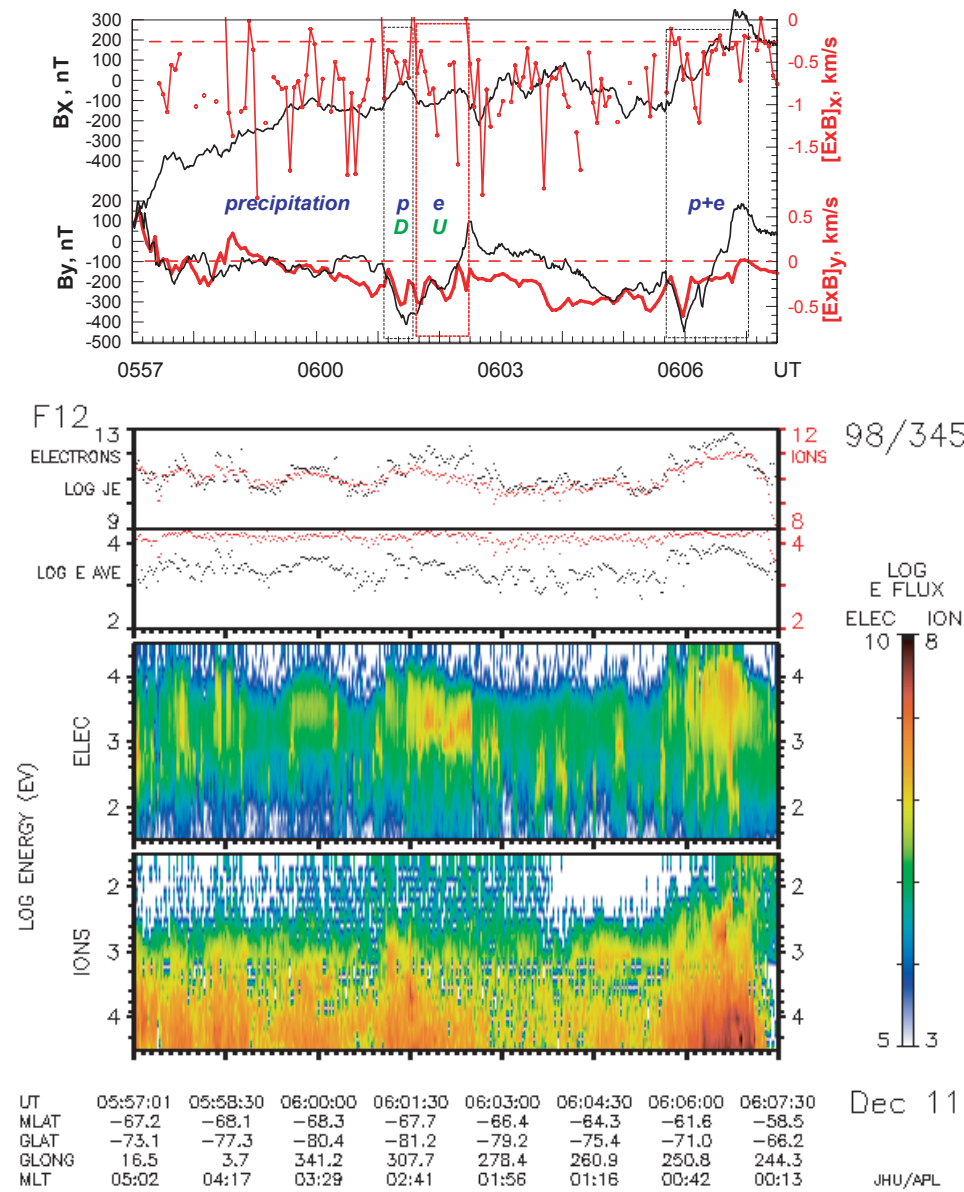

Fig. 4. Summary of DMSP F12 observations during the streamer crossings at 06:01 UT and 06:05 UT.

some signatures of upward FA current could be noticed, but it does not show the 1-D properties. In this case the type II precipitation is also seen on the dusk side of the equatorward convection region.

The next event (Fig. 4) is similar in that there were again the crossings of two streamers. The first crossing of an intensified streamer near the poleward oval boundary in the early morning sector displays some mixture of the two types of characteristics. Here the precipitation is clearly increased in both ions and electron components between 06:01:27 and 06:02:30 UT, however, the proton (electron) energy flux was preferentially enhanced in the dawn-side (dusk-side) half in association with downward (upward) FA currents. The convection flow has a considerable equatorward component but it is difficult to decide on whether the convection jet could be inferred in this case. (The $V_{x}$ component displays an intense eastward convection component expected in the westward electrojet region, but it is very noisy, uncorrelated with $D B_{x}$ and is not used in the detailed analysis.)

The second crossing has the type II properties, actually here the streamer merges with the regular equatorward diffuse precipitation and it is not easy to distinguish between them. The Polar UVI images show that the intrusion of two streamers into the equatorward oval occurred about 05:50 UT in the sector near 01:00 MLT. It was the intensified diffuse precipitation associated with this intrusion that actually controlled the emission distribution in this portion of the equatorward oval. There is also a strong (mostly upward) fieldaligned current over the structure which is situated at the westward flank of the stream of equatorward convection.

The last example includes the streamer observation in its late stage of life. In Fig. 5 two DMSP spacecraft (F13 and F14) crossed the poleward half of what is usually called a torch-like structure (e.g. Akasofu, 1974). It was born by the streamer which activated in the poleward oval at 06:08 UT and intruded into the equatorward oval at 06:12 UT at the meridian 01:50 MLT. The resulting structure persisted for next $10-15 \mathrm{~min}$, drifting eastward until its registration at 06:26 UT at 02:50 MLT. It could then be tracked in time until at least 06:50 UT, drifting towards the 04:00 MLT meridian. Although this structure was crossed in its poleward part $\left(-67^{\circ}\right.$ CGLat.) i.e. rather far from equatorward boundary of the oval (at $\sim 7^{\circ}$ according to F14 crossing made at midnight), it clearly displays the type II properties with both proton and electron fluxes enhanced simultaneously reaching to the level comparable to that in the equatorward oval. 

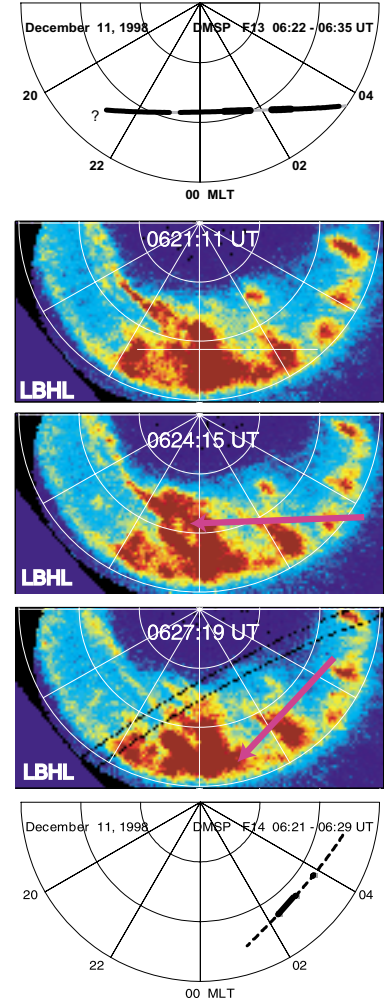
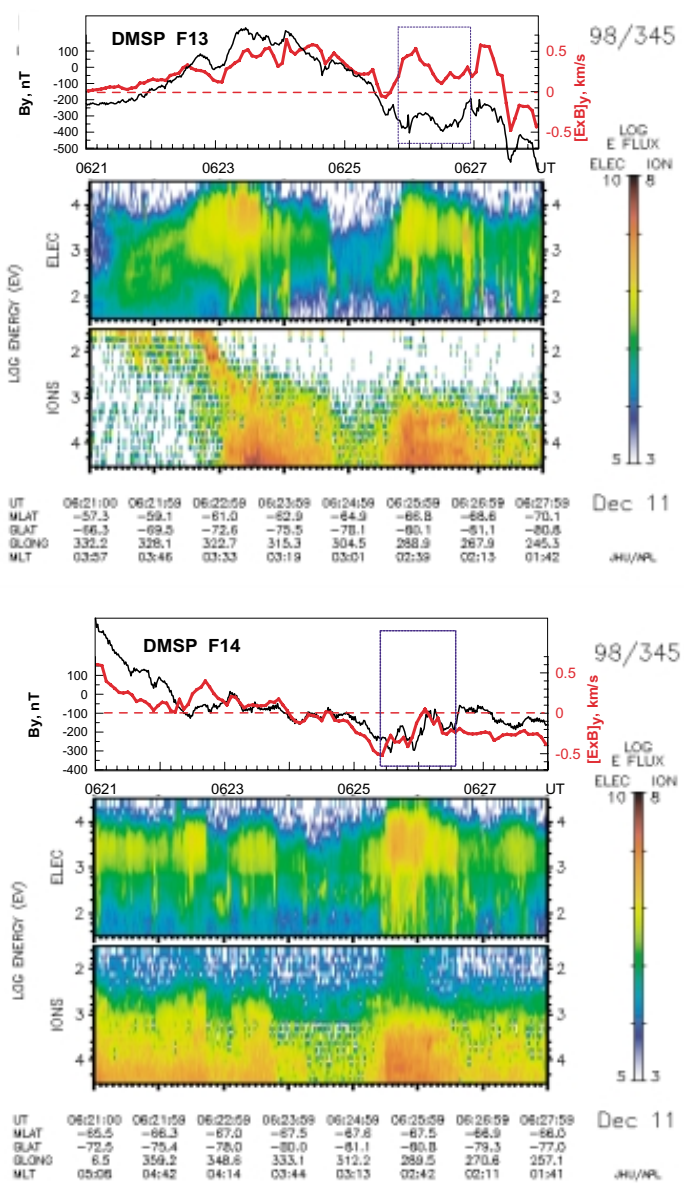

Fig. 5. Summary of DMSP F13/F14 observations during the crossing of touch-like structure (old streamer) at $\sim 06: 26$ UT.

\subsection{Summary of observations}

We have typically found a good agreement between the precipitation patterns seen by the DMSP SSJ4 spectrometer and the auroral patterns (along the DMSP trajectory mapped into the northern ionosphere) observed by Polar UVI. This agreement particularly follows from the cases with the most (dynamically and structurally) simple auroral patterns which are shown in Figs. 1 to 4 . The shifts of conjugate points within $2^{\circ}$ of CGL at and of 00:50 MLT are usually sufficient to obtain agreement between the observations in the two hemispheres. With this remark we can speak about the conjugacy of the auroral streamers.

A dozen well-identified streamers have been processed. Their parameters are summarized in Table 1, which requires a few comments. The UT of precipitation onset and its duration in the third column corresponds to the accelerated electrons if they are the Type I events, but it corresponds to the width of enhanced proton+electron precipitation in Type II events. Its spatial size $L_{x}$, computed from the crossing duration (using $v_{x}=6.8 \mathrm{~km} / \mathrm{s}$, thereafter all sizes are scaled to $\mathrm{h}=110 \mathrm{~km}$ in the ionosphere), gives us the upper estimate of the spatial scale across the structure. The upward FAC density in Table 2 gives the lower estimate of the average $j_{\|}$(be- ing averaged over the structure and using the upper estimate $L_{x}$ ). The spatial scale of the convection stream (convection jet) and resulting potential drop were determined based on the width of the whole (downward+upward) FAC sheet (for type I events), or the width of the whole precipitation pattern (for type II events) if the convection jet was not well expressed. The regression coefficient $B_{y} / V_{y}$ over that region characterizing the coupling efficiency between the magnetosphere and the ionosphere is given only if the correlation between these parameters was high enough $(R>0.7$, the value is given in parenthesis). A summary of the observational results is as follows.

The five Type I events are characterized by the intense electron precipitation (with signatures of field-aligned acceleration) associated with depressed (or unchanged) proton precipitation, which coincides with the region of strong (above a few $\mu \mathrm{A} / \mathrm{m}^{2}$ ) upward FAC. This FAC sheet always has an adjacent sheet of downward FAC of nearly equal magnitude situated at the more eastward location (like in the scheme in Fig. 1). This double sheet FAC pattern either coincides (like in NN1, 2 in the Table 1) with the stream of equatorward convection or it is found inside the wide equatorward convection (NN5, 7, 11), its maximal magnitude (only $V_{y}$ component used) changes between 0.5 and $1.2 \mathrm{~km} / \mathrm{s}$. The 
Table 1. Characteristics of auroral streamers and streamer-related precipitation observed during 11 December 1998 SMC event.

\begin{tabular}{|c|c|c|c|c|c|c|}
\hline $\mathrm{NN}$ & $\mathrm{SC}$ & $\begin{array}{l}\text { e-precipitation, } \\
\text { UT start plus } \\
\text { duration }\end{array}$ & CGLat / MLT & $L_{x}, \mathrm{~km}$ & Type & $\begin{array}{l}\text { Streamer life-time and dynamics as } \\
\text { observed by Polar UVI }\end{array}$ \\
\hline 1 & $\mathrm{f} 12$ & $02: 38: 32+12 \mathrm{~s}$ & $68^{\circ} / 23.7$ & 80 & I & $\begin{array}{l}>5 \text { min, could be a new activation of old } \\
\text { streamer structure }\end{array}$ \\
\hline 2 & $\mathrm{f} 14$ & $03: 01: 28+22 \mathrm{~s}$ & $69^{\circ} / 00.5$ & 150 & I & Since 02:58 UT (N1) \\
\hline 3 & $\mathrm{f} 13$ & $03: 02: 45+90$ & $67^{\circ} / 23.5$ & 615 & II & Since 02:58 UT (N2) \\
\hline 4 & $\mathrm{f} 13$ & $03: 04: 45+15$ & $67^{\circ} / 22.7$ & 100 & II & Since 02:57 UT (N3) \\
\hline 5 & $\mathrm{f} 13$ & $04: 48: 30+37$ & $67^{\circ} / 23$ & 250 & I & Activated since $04: 46$ UT \\
\hline 6 & $\mathrm{f} 13$ & $04: 50: 45+60$ & $64^{\circ} / 21.5$ & 410 & II & Since 04:39 UT \\
\hline 7 & $\mathrm{f} 12$ & $06: 01: 27+60$ & $68^{\circ} / 02.7$ & 410 & I & $\begin{array}{l}\text { Two streamers developed since 05:53 and } \\
05: 59 \text { UT }\end{array}$ \\
\hline 8 & $\mathrm{f} 12$ & $06: 05: 40+80 \mathrm{~s}$ & $62^{\circ} / 00.7$ & 540 & II & $\begin{array}{l}\text { EQ part of old streamer, } \\
\text { visible since 05:53 UT }\end{array}$ \\
\hline 9 & $\mathrm{f} 14$ & $06: 25: 33+60 s$ & $67^{\circ} / 02.7$ & 410 & II & $\begin{array}{l}\text { Touch structure, developed since 06:08 UT, } \\
\text { intruded to EQ oval at 06:12 UT, }\end{array}$ \\
\hline 10 & $\mathrm{f} 13$ & $06: 25: 50+60 \mathrm{~s}$ & $67^{\circ} / 02.6$ & 410 & II & $\begin{array}{l}\text { (cont.), then drift eastward by } 1 \mathrm{~h} \mathrm{LT} \text {. Seen } \\
\text { until at least 06:50 UT (at 04:00 LT) }\end{array}$ \\
\hline 11 & $\mathrm{f} 12$ & $11: 17: 53+6 s$ & $63^{\circ} / 23.1$ & 40 & I & $\begin{array}{l}\text { Activated at } \sim 11: 13 \text { UT at the place of old } \\
\text { streamer (existing since 10:49 UT) }\end{array}$ \\
\hline
\end{tabular}

upward FAC and precipitation of field-aligned accelerated electrons occupies the dusk flank of the convection stream. This streamer-associated convection jet is not strong, showing typically a few $\mathrm{kV}$ potential drop, with the maximal value $13 \mathrm{kV}$ found in the strongest event (N11, Fig. 2). The cross-B magnetic variations often show a linear polarization, indicating a sheet geometry, with the inferred FAC sheet orientation being nearly aligned along the streamer (see, e.g. Figs. 1 and $3)$. The $B_{y}$ and $V_{y}$ variations typically show a nice correlation, indicating a strong coupling between the convection and the FAC, their regression coefficient $\left(B y=a V_{y}+b\right)$ is in the range $a \simeq 300-400 \mathrm{nT} /(\mathrm{km} / \mathrm{s})$ in the four cases shown in Table 2.

The spatial scale across the type I streamer (electron precipitation and upward FAC) ranges between $40-80 \mathrm{~km}$ in the intense narrow streamers (NN 1 and 11, Figs. 1 and 2) and may reach 250 to $450 \mathrm{~km}$ in the cases of relatively wide structured streamer formations. (The latter ones may rather correspond to the multiple superimposed narrow streamers, see, e.g Figs. 3 and 4, although we cannot address this aspect in more detail based on the data available.) The spatial scale of the convection jet (and/or double sheet FAC structure) ranges between 150 and $400 \mathrm{~km}$.

Six crossings over the type II streamer-associated precipitation structures have been also analysed. The association with the streamer-aligned equatorward convection and fieldaligned currents is more variable in this group. There are cases with clear FAC localized in the same region (like N8, Fig. 4) or examples with no such association (although with some FAC embedded, like in N3, 4). The last two events have been embedded in the broad region of equatorward convec- tion and coincided with the broad peaks of convection velocity, whereas in some other events (NN6, 8, 9, see Figs. 3, 4, and 5) the precipitation was observed at the westward flank of equatorward convection like it was in the type 1 events. (The strong noise in the registered $V_{x}$ flow velocity prevent us from discussing the association with the convection in more detail.)

The latitudinal dependence of type I, II events is quite well organized in the sense that only type I events were observed if the streamers were crossed near the poleward oval boundary $(\mathrm{NN} 1,2,11)$, whereas only type II events were seen where the streamer contacts with the equatorward oval (N8 and some other cases not included in this study). Sometimes both types could be seen in the middle of auroral oval at latitudes as low as $63^{\circ}$ CGLat for type I event (N11, Fig. 2) and at latitudes as high as $67^{\circ}-68^{\circ}$ CGLat for type II events (N3, $4,9)$. In fact, there are actually some cases with coexistent features of both types. Examples could be event 7 in Fig. 4 . Whereas the spatial separation of proton and electron precipitation around 06:01:30 UT, as well as the narrow energy peak on the spectrogram (evidence of field-aligned acceleration) are seen here, the proton precipitation is clearly enhanced inside of the area of electron precipitation (although not as much as it was between 06:01:10 and 06:01:30 UT). We emphasize that our categorization to two types is an idealization, and that there could be a number of events showing different mixtures of both categories. 


\section{Plasma Sheet Bubble and its lonospheric Mapping}

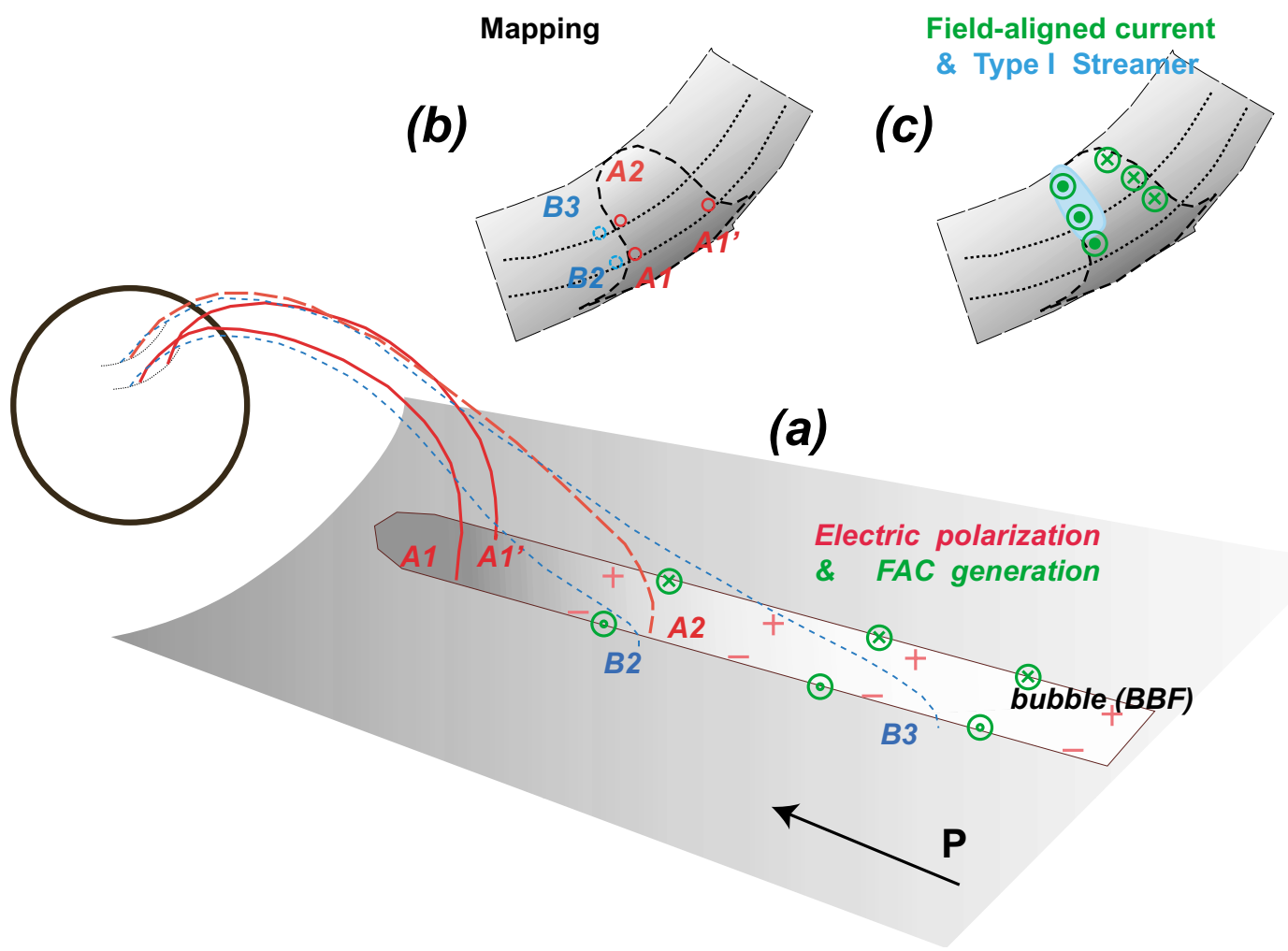

Fig. 6. Scheme showing the ionospheric mapping and manifestations of the narrow bursty bulk flow as predicted by the plasma bubble model.

\section{Discussion}

Figure 6 provides a schematic summary of our findings (Fig. 6c), together with the diagrams to illustrate the streamer relationship with the bursty bulk flows (Fig. 6a), as well as the mapping issues (Fig. 6b). In Fig. 6c the type I streamer precipitation is shown in the region of strong upward FAC located at the western flank of the structure which also includes the downward FAC at its eastern flank. The convection is expected to be in the equatorward direction (mostly along the streamer, that is with south-west electric field in the Northern Hemisphere for our events), although the spatial distribution of convection is a matter of some ambiguity, as will be discussed later. Such pattern has considerable support from the previous studies.

The most comprehensive investigation of a 3-D current system associated with a NS-aligned auroral structure has been made by Amm et al. (1999), who used the radar electric field observations and the magnetometer network data to reconstruct the distributions of the conductivities and currents. Besides the strong upward FAC sheet $\left(j_{\max } \sim 25 \mathrm{~A} / \mathrm{km}^{2}\right)$ nearly collocated with the $100-\mathrm{km}$ wide north-south auroral structure, they also inferred the downward FAC sheet (up to $15 \mathrm{~A} / \mathrm{km}^{2}$ ) at the eastern side of entire $250 \mathrm{~km}$ wide structure, as well as the south-west electric field of $20-30 \mathrm{mV} / \mathrm{m}$ (possibly the lower limit), corresponding to the $>0.5 \mathrm{~km} / \mathrm{s}$ convection along the streamer. These patterns and scale-sizes are consistent with those found in our analysis, with the exception of a few times larger current density of field-aligned currents. This difference could be partly because our $j_{\|}$numbers provide the lower estimates, and partly because the Amm et al. observations were made during a very disturbed event ( $>1000 \mathrm{nT}$ in total $A E$ index, $\sim 500 \mathrm{nT}$ magnetic variations associated with the streamer, and $\sim 200 \mathrm{~S}$ conductivity in the auroral streamer).

The auroral streamers are observed in the middle of the auroral oval, that is on the closed field lines of the tail plasma sheet, which is consistent with the apparent conjugacy of streamer-related precipitation found in our study. Given that location, one may attempt to associate the streamer-related equatorward convection jet and field-aligned currents with the narrow streams of the earthward convection in the plasma sheet, that is with the bursty bulk flows (BBFs). Such association has been directly confirmed in the detail comparisons of auroral dynamics and plasma sheet convection (e.g. Lyons et al., 1999; Sergeev et al., 2000; Nakamura et al., 2001a). Particularly valuable are the results by Nakamura et al. (2001b), who studied statistically strong short-duration isolated BBFs 
Table 2. Streamer-related field-aligned currents and convection (continuation of Table 1).

\begin{tabular}{cccll} 
NN & Type & FAC $d B / d T, j \|$ & $\begin{array}{l}\text { Convection jet: } \\
\text { crossing time } \\
\Delta T, \mathrm{~s} / \Delta \Phi, \mathrm{kV}\end{array}$ & $\begin{array}{l}B_{y} / V_{y}, \\
\mathrm{nT} /(\mathrm{km} / \mathrm{s}) ; \\
(\mathrm{R})\end{array}$ \\
\hline 1 & I & $390 \mathrm{nT} / 10 \mathrm{~s} ; 5.3 \mu \mathrm{A} / \mathrm{m}^{2}$ & $25 \mathrm{~s} / 3 \mathrm{kV}$ & $420(0.93)$ \\
2 & I & $370 \mathrm{nT} / 24 \mathrm{~s} ; 2.1 \mu \mathrm{A} / \mathrm{m}^{2}$ & $20 \mathrm{~s} / 3 \mathrm{kV}$ & $320(0.7)$ \\
3 & II & - & $-13 \mathrm{kV}^{(2)}$ & - \\
4 & II & - & $-/ 3 \mathrm{kV}^{(2)}$ & - \\
5 & I & $551 \mathrm{nT} / 31 \mathrm{~s} ; 2.5 \mu \mathrm{A} / \mathrm{m}^{2}$ & $>60 \mathrm{~s} / 11 \mathrm{kV}^{(2)}$ & $374(0.90)$ \\
6 & II & - & $>60 \mathrm{~s} / 1.5 \mathrm{kV}$ & $550(0.93)$ \\
7 & I & $510 \mathrm{nT} / 61 \mathrm{~s} ; 1.2 \mu \mathrm{A} / \mathrm{m}^{2}$ & $-/ 5 \mathrm{kV}$ & - \\
8 & II & Embedded intoR $2 \mathrm{FAC}$ & $-/ 3 \mathrm{kV}$ & $850(0.77)$ \\
9 & II & - & $60 \mathrm{~s} / 3.5 \mathrm{kV}$ & - \\
10 & II & - & $60 \mathrm{~s} / 5.5 \mathrm{kV}$ & - \\
11 & I & $410 \mathrm{nT} / 8 \mathrm{~s} ; 7 \mu \mathrm{A} / \mathrm{m}^{2}$ & $50 \mathrm{~s} / 13 \mathrm{kV}$ & $310(0.75)$ \\
\hline
\end{tabular}

${ }^{(1)}$ Results are given only if correlation coefficient is high, $R>0.7$

${ }^{(2)}$ Potential drop across the electron precipitation region or up/down FAC pair (convection jet is not clearly defined)

accompanied by isolated (thus, well identified) auroral signatures. After determining which BBF flank was crossed by the Geotail spacecraft, and performing the spacecraft mapping into the ionosphere with event-adjusted magnetospheric models, they were able to show that BBF-associated structures (both localized brightenings and auroral streamers) are conjugate to the mappings from the western flank of the BBF. The mappings from the dawn-flank crossings were systematically displaced eastward of the auroral structure, suggesting that auroral streamers show us only a limited part of the entire BBF plasma stream, whose ionospheric projection has a spatial scale about 00:50-01:00 MLT (or 300-600 km in E-W direction). This picture and scales are consistent with our results. Localization of accelerated electrons (type I events in our classification) on the westward side of the structure (which is always true for type I events) could be understood as the result of an upward field-aligned electric field which is localized in the upward FAC region. The estimated amplitudes of upward FACs (exceeding a few $\mu \mathrm{A} / \mathrm{m}^{2}$, see Table 2) are in the range where the $\mathrm{kV}$-range field-aligned potential drops are expected from the Knight relationship (e.g. Lyons, 1980). Finally, the occurrence of the upward FACs at the westward flank of BBF projection to the ionosphere is a consequence of the current continuity feature if we either map the strip of enhanced westward electric field into the ionosphere (quasi-static mapping) and/or if we map into the ionosphere the field-aligned currents generated at the flanks of the equatorial plasma sheet stream. In either case the region I sense FAC pattern is expected, and this is what is typically observed.

The appearance of the type II streamer-related precipitation is not as easy to explain. Although the plasma should be heated and compressed in the earthward-contracting BBF flux tubes, the observations actually show that the energy flux and the plasma pressure in the BBF was smaller than it was before the event, or than it is in the surrounding flux tubes. This is known as a "bubble" property of the bursty bulk flows (since Sergeev et al., 1996b and was established in many statistical studies of the BBF events in the central plasma sheet, e.g. Kauristie et al., 2000; Schodel et al., 2001; Nakamura et al., 2001b). In physical terms, this is a known property of the interchange instability in which only a flux tube with reduced plasma content can slip earthward with respect to its neighbour. This property (reduced plasma content, $p V^{\gamma}$, with the flux tube volume $V$, plasma pressure $p$ and $\gamma=5 / 3$ ) is a necessary condition to obtain the BBF-related plasma element polarized, as shown in Fig. 6a, with an additional electric field directed from dawn to dusk, implying the fast earthward flow in the stream. The required property is expected to be provided by the reconnection process. This "bubble model" of the BBFs developed by Chen and Wolf (1993, 1999) predicts that such a plasma stream will move earthward (with plasma pressure increasing) until its plasma content will match the plasma content of the surrounding tubes. These variations are schematically shown with variable shading in the Fig. 6 a.

At first glance the pressure and energy flux variations across the bubble and across the type 2 streamer look opposite, but this inconsistency could be resolved by taking into account the deformation of the magnetic configuration by the field-aligned currents generated by the bubble. Chen and Wolf (1993) noticed that the field lines inside the bubble are dipolarized as compared to the surrounding tubes, therefore, the mapping is deformed, as sketched in Figs. $6 a$, b. Two neighbour equatorial points $\mathrm{A} 2$ (inside the BBF) and B2 (outside the BBF) are mapped to different latitudes in the ionosphere, with A2 appearing at higher latitude than B2 (Fig. 6b). Such deformation implies that the spacecraft 
traversing the streamer boundary (along the dotted line in Fig. 6b) will cross the points $\mathrm{B} 2$ and $\mathrm{A} 1$, where $\mathrm{A} 1$ has the equatorial foot at smaller distance than B2 has, that is in the region of larger pressure than $\mathrm{A} 2$ and (potentially larger) than B2. In this way the opposite gradients could form across the convection jet (bubble, or BBF) boundaries in the ionosphere as compared to the plasma sheet profile.

The field-aligned currents' pattern involved and the mapping deformations produced are similar to those responsible for the formation of the auroral bulge during the substorm expansion phase (e.g. Vasilyev et al., 1986). With the typical FAC-associated magnetic shear observed during the streamer crossing, $d B \sim 400-500 \mathrm{nT}$ (Table 2), the associated sheet current density (per unit length) is approximately $J \sim 450 \mathrm{~A} / \mathrm{km}$. With the length of this FAC sheet (length of the streamer) often approaching (and exceeding) $1000 \mathrm{~km}$, one has the total FAC about $0.5 \mathrm{MA}$ in one hemisphere. Such a current is capable of shifting the footpoint very significantly (up to $5^{\circ}$ CGLat for the $0.5 \mathrm{MA}$ substorm current wedge current, according to Fig. 2 of Vasyliev et al., 1986). Although some numerical exercises computing the magnetic deformation were reported by Chen and Wolf (1993, 1999), the model was not very realistic, so a future task is to compute the bubble-related configuration capable of reproducing the observed increases of plasma pressure in type II streamer-related precipitation.

The streamer-associated convection pattern also requires some comments. Although we had only one $\left(V_{y}\right)$ horizontal flow component reliably observed, there were many cases where the convection jet is not well-defined or the type II precipitation band (or the whole up+down FAC system in type I events) does not coincide with the entire stream of equatorward convection, as would follow from a naive mapping of the equatorial convection stream into the ionosphere (see also Amm et al., 1999 results). Also, rather small values of the potential drop associated with convection streams in the ionosphere (a few $\mathrm{kV}$, maximal value about $13 \mathrm{kV}$, see 8 th column of Table 1) look difficult to reconcile with the conclusions that strong BBF events have an "effective potential drop" $(E y \cdot L y)$ of a few tens $\mathrm{kV}$ or larger (Sergeev et al., 2000). Several factors will complicate and modify the ionospheric appearance of the convection streams. (1) Significant mapping deformations (with the neighbouring points at the boundary mapped to very different distances, like points A1 and B2 in Fig. 6) could seriously affect the result. (2) The transient character of the BBF (with flow speed comparable with the speed of fast and Alfvénic waves in the plasma sheet) make the propagation effects essential (e.g. Chen and Wolf, 1999). The major consequence is that a considerable part of the electric field is of a inductive character, which is not easily mapped to the ionosphere. (3) A number of processes may modify the electric field pattern even when dealing with the electrostatic mapping. For example, the fieldaligned potential drops above the electron acceleration regions may modify the electric field distributions (and shortcircuit a part of the potential drop) on the short scales, less than or about $100 \mathrm{~km}$, as recently discussed by Borovsky and
Bonnell (2001).

The ratio between the highly correlated $y$-components of the convection and the magnetic variation (last column in Table 1) allows us to evaluate some properties of the electric coupling process. It shows the values $300-400\left(\mathrm{nT}^{*} \mathrm{~s}\right) / \mathrm{km}$ for the type I streamer precipitation, which corresponds to an effective integral conductivity $\delta B /\left(\mu_{0} \delta E\right) \simeq 6-8 \mathrm{~S}$. This value is much larger than the Alfvén wave impedance values usually cited (0.5 S, e.g. Lysak, 1990), but is comparable to the expected ionospheric conductivity (the streamers were traversed in the sunlit hemisphere under solar zenith angles about $70^{\circ}-80^{\circ}$, so that the background $\Sigma \sim 2-3 \mathrm{~S}$ can be expected due to solar EUV illumination, e.g. Moen and Brekke, 1993, with additional input from particle precipitation). Such values point to the quasi-static coupling which exist in the streamer-related flux tubes.

\section{Conclusions}

This study provides the parameters of streamer-related convection, field-aligned currents and precipitation, which are important for addressing the generation mechanisms of auroral streamers and constraining the corresponding models.

1. Observational data confirm the association of auroral streamers with equatorward convection jets, which, however, are not as strong (several $\mathrm{kV}$ ) as the intense plasma sheet BBFs, and, probably are not the direct quasi-static electrostatic mapping of the BBF-related narrow plasma streams in the equatorial plasma sheet.

2. Signatures of field-aligned electron acceleration and direct association with the sheets of strong (exceeding a few $\mu \mathrm{A} / \mathrm{m}^{2}$ ) upward field-aligned current in type I events directly confirm that auroral streamers (at least in their poleward half) are formed by the field-aligned electron acceleration in the strong upward field-aligned current. The entire pattern is consistent with this upward FAC generated at the dusk flank of the narrow plasma sheet convection stream.

3. An unpredicted observation is that of the type II precipitation (in the equatorial half of the streamer) which shows enhanced energy flux (and energy density) of both protons and electrons. We argue that it is formed by the mapping between magnetosphere and ionosphere which is strongly deformed by the strong (several tenths MA) streamer-related field-aligned currents, in much the same way as the auroral bulge is formed during the substorms.

Acknowledgements. The authors thank D. H. Fairfield for his detailed comments on the manuscript. This work by V. A. Sergeev has been supported by RFBR grant 03-02-17533 and Intergeophysics Program.

Topical Editor T. Pulkkinen thanks D. H. Fairfield and another referee for their help in evaluating this paper. 


\section{References}

Akasofu, S.-I.: A study of auroral displays photographed from DMSP-2 satellite and from the Alaskain meridian chain of stations, Space Sci. Rev., 16, 617-725, 1974.

Amm, O., Pajunpaa A., and Brandstrom, U.: Spatial distribution of conductances and currents associated with a north-south auroral form during a multiple-substorm period, Ann. Geophysicae 17, $1385,1999$.

Amm, O. and Kauristie, K.: Ionospheric signatures of bursty bulk flows, Surveys in Geophysics, 23, 1-32, 2002.

Angelopoulos, V., Baumjohann, W., Kennel, C. F., Coroniti, F. V., et al.: Bursty bulk flows in the inner central plasma sheet, J.Geophys, Res., 97, 4027, 1992.

Angelopoulos, V., Phan, T. D., Larson, D. E., Mozer, F. S., et al.: Magnetotail flow bursts: association to global magnetospheric circulation, relationship to ionospheric activity and direct evidence for localization, Geophys.Res.Lett., 24, 2271, 1997.

Baumjohann, W.: The near-Earth plasma sheet: An AMPTE/IRM perspective, Space Sci. Rev, 64, 141-191, 1993.

Borovsky J. and Bonnell, J. W.: The DC electric coupling of flow vortices and flow channels in the magnetosphere to the resistive ionosphere, J. Geophys. Res., 106, 28 967-28 988, 2001.

Boyle, C. B., Reiff, P. H., Hairston, M. R.: Empirical polar cap potential, J. Geophys. Res, 102, 111-128, 1997.

Chen, C. X. and Wolf, R. A.: Interpretation of high-speed flows in the plasma sheet, J. Geophys. Res., 98, 21 409-21 423, 1993.

Chen, C. X. and Wolf, R. A.: Theory of thin-filament motion in Earth's magnetotail and its application to bursty flows, J. Geophys. Res., 104, 14 613-14 628, 1999.

Fairfield, D. H., Mukai, T., Brittnacher, M., Reeves G. D., et al.: Earthward flow bursts in the inner magnetotail and their relation to auroral brightenings, AKR intensifications, geosynchronous particle injections and magnetic activity, J. Geophys. Res., 104, 355, 1999.

Ieda, A., Fairfield, D. H., Mukai, T., Saito, Y., et al.,: Plasmoid ejection and auroral brightenings, J. Geophys. Res., 106, 38453857, 2001.

Ieda, A., Shue, J.-H., Liou, K., Ohtani, S.-I., et al.: Quiet time magnetotail plasma flow: Coordinated Polar ultraviolet images and Geotail observations, J. Geophys. Res., Vol. 108, No. A9, 13451357, 10.1029/2002JA009739, 2003.

Kauristie, K., Sergeev, V. A., Kubyshkina, M., Pulkkinen, T. I., et al.: Ionospheric current signatures of transient plasma sheet flows, J. Geophys. Res., 105, 10 677-10 688, 2000.

Liou, K., Newell, P. T., Meng, C.-I., Brittnacher, M., and Parks, G.: Synoptic auroral distributions: a survey using Polar ultraviolet imagery, J. Geophys. Res., 102, 27 197-27 207, 1997.

Lyons, L. R.: Generation of large-scale regions of auroral currents, electric potentials, and precipitation by the divergence of convection electric field, J. Geophys.Res., 85, 17-30, 1980.

Lyons, L. R., Nagai, T., Blanchard, G. T., Samson, J. C., et al.: Association between Geotail plasma flows and auroral poleward boundary intensifications observed by CANOPUS photometers,
J. Geophys. Res., 104, 4485-4497, 1999.

Lyons, L. R., Zesta, E., Xu, Y., and Sanchez, E. R., et al.: Auroral poleward boundary intensifications and tail bursty flows: A manifestation of a large-scale ULF oscillation? 10.1029/2001JA00024, 2002.

Lysak, R. L.: Electrodynamic coupling of the magnetosphere and the ionosphere, Space. Sci. Rev., 52, 33-88, 1990.

Moen, J. and Brekke, A.: The solar flux influence on quiet time conductances in the auroral ionosphere, Geophys. Res. Lett., 20, 971-974, 1993.

Nakamura, R., Baumjohann, W., Brittnacher, M., Sergeev, V. A., et al.: Flow bursts and auroral activations: Onset timing and foot point location, J. Geophys. Res., 106 , 10777-10 790, 2001a.

Nakamura, R., Baumjohann, W., Schodel, R., Brittnacher, M., et al.: Earthward flow bursts, auroral streamers, and small expansions, J. Geophys. Res., 106 , $10791-10$ 804, 2001b.

Newell, P. T. and Meng, C.-I.: Low-altitude observations of dispersionless substorm injections, J. Geophys. Res., 92, 10063 $10072,1987$.

Rich, F. J., Hardy, D. A., Gussenhoven, M. S.: Enhanced ionosphere-magnetosphere data from the DMSP satellites, EOS Trans. AGU, 66, 513, 1985.

Schodel, R., Nakamura, R., Baumjohann, W., Mukai, T.: Rapid flux transfer and the plasma eheet reconfiguration, J. Geophys. Res., 106, 8381-8396, 2001.

Senior, C., Cerisier, J.-C., Rich, F., Lester, M., Parks, G.: Strong sunward propagating flow bursts in the night sector during quiet solar wind conditions: SuperDARN and satellite observations, Ann. Geophys., 20, 771-779, 2002.

Sergeev, V. A., Pellinen, R. J., Pulkkinen, T. I.: Steady magnetospheric convection: a review of recent results, Space Sci. Rev., 75, 551-604, 1996a.

Sergeev, V. A., Angelopoulos, V., Cattell, C. A., Russell, C. T.: Detection of localized plasma bubbles in the plasma sheet, J. Geophys. Res., 101, 10817-10 826, 1996b.

Sergeev, V. A., Sauvaud, J.-A., Popescu, D., Kovrazhkin, R. A., et al.: Multiple-spacecraft observation of a narrow transient plasma jet in the Earth's plasma sheet, Geophys.Res, Lett., 27, 851-854, 2000.

Sergeev, V. A., Kubyshkina, M. V., Liou, K., Mukai, T., et al.: Substorm and convection bay compared: Auroral and magnetotail dynamics during convection bay, J. Geophys. Res., 106, 18843 $18855,2001$.

Sergeev V. A.: Ionospheric signatures of magnetospheric particle acceleration in substorms - How to decode them?, Proc.6th Intern. Conf. on Substorms, Univ. of Washington, Seattle, 39-46, 2002.

Serizawa, Y. and Sato, T.: Generation of large scale potential difference by currentless plasma jets along the mirror field, Geophys. Res. Lett., 11, 595-599, 1984.

Vasyliev, E. P., Sergeev, V. A., Malkov, M. V.: Three-dimensional effects of Birkeland current loop, Geomagn. Aeronomy, 26, 1, 114-118, 1986. 